\title{
Seeing two faces together: preference formation in humans and rhesus macaques
}

David Méary ${ }^{1}$, Zhihan $\mathrm{Li}^{3}, \mathrm{Wu} \mathrm{Li}^{3}$, Kun Guo ${ }^{2}$ and Olivier Pascalis ${ }^{1}$

(1)

Laboratoire de Psychologie et Neurocognition, CNRS UMR 5105, Université Grenoble Alpes, 38040 Grenoble Cedex 9, France

(2)

School of Psychology, University of Lincoln, Lincoln, LN6 7TS, UK

(3)

State Key Laboratory of Cognitive Neuroscience and Learning and IDG/McGovern Institute for Brain Research, Beijing Normal University, Beijing, 100875, China

David Méary (Corresponding author)

Email: david.meary@upmf-grenoble.fr

Olivier Pascalis

Email: olivier.pascalis@upmf-grenoble.fr

Received: 28 June 2013Revised: 13 February 2014Accepted: 3 March 2014Published online: 18 March 2014

Abstract

Humans, great apes and old world monkeys show selective attention to faces depending on conspecificity, familiarity, and social status supporting the view that primates share similar face processing mechanisms. Although many studies have been done on face scanning strategy in monkeys and humans, the mechanisms influencing viewing preference have received little attention. To determine how face categories influence viewing preference in humans and rhesus macaques (Macaca mulatta), we performed two eye-tracking experiments using a visual preference task whereby pairs of faces from different species were presented simultaneously. The results indicated that viewing time was significantly influenced by the pairing of the face categories. Humans showed a strong bias towards an own-race face in an Asian-Caucasian condition. Rhesus macaques directed more attention towards non-human primate faces when they were paired with human faces, regardless of the species. When rhesus faces were paired with faces from Barbary macaques (Macaca sylvanus) or chimpanzees (Pan troglodytes), the novel species' faces attracted more attention. These results indicate that monkeys' viewing preferences, as assessed by a visual preference task, are modulated by several factors, species and dominance being the most influential.

Electronic supplementary material 
The online version of this article (doi:10.1007/s10071-014-0742-3) contains supplementary material, which is available to authorized users.

Keywords

HumansRhesus macaquesPreferencesFacesEye-tracking

\section{Introduction}

Viewing preferences have been studied extensively in human adults, infants, and non-human primates, but the processes leading to increased or decreased looking time towards one out of two, or more, stimuli presented simultaneously are difficult to circumvent. The novelty or familiarity of the visual stimulus is often put forward to explain differences in viewing time (Fantz 1964) but the cognitive processes by which these two general aspects influence viewing behavior are still subject to debate (Park et al. 2010). Viewing preferences result from the interplay of multiple factors. The properties of the viewed object such as symmetry (Enquist and Arak 1994) or color (Taylor et al.

2013) and other cognitive, social, and emotional factors, as illustrated by the effects of mere exposure (Zajonc 1968), affects (Bornstein 1989) or category learning (Rentschler et al. 1999), also contribute to shaping visual preferences. The list is far from being complete and, given its complexity, it is reasonable to simplify the analysis of preferences by focusing on one type of visual object. Because faces are important for both human and non-human primates, they are well suited for studying preference formation and could help in identifying the peculiarities of human and nonhuman primates' social cognition.

Face processing is often associated with expertise, meaning that a familiar face is identified at the level of the unique individual (e.g., Bob, Mary) rather than categorized at the more generic level of race or gender (Tanaka 2001). The cognitive process underlying face expertise is thus classically described as a hierarchical categorization process (Bruce and Young 1998). Categorization of faces according to facial features can be seen from the first months of human infants' development. For example, 3 month-olds categorize faces into own versus other species (Heron-Delaney et al. 2011; Di Giorgio et al. 2013), European versus Asian faces (Kelly et al. 2005), and male versus female (Leinbach and Fagot 1993; Quinn et al. 2002). When infants are presented with a pair of faces from these different categories presented side-by-side, they tend to look longer at the faces belonging to their own species and own race, and at the faces of their caregiver's gender. These findings suggest that looking preferences during infancy are influenced by category-specific knowledge acquired through selective experience, that is, familiarity. However, while stimulus familiarity best predicts infants' looking behavior in a visual preference task, novelty attracts infants' attention when they are first familiarized with a stimulus, as illustrated by the effect of habituation (Fantz $\underline{1964}$ ).

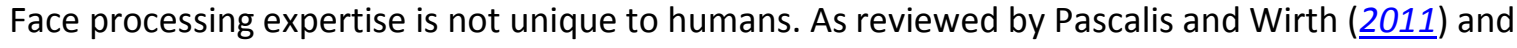
Parr (2011), non-human primates also show experience-related looking preferences. Using a selfcontrolled single face presentation task, Fujita (1987) found that macaques from five different species preferred to look at their conspecifics' faces rather than at the faces of other macaque species, suggesting that conspecificity was guiding viewing preferences. Using a visual preference task, Sugita (2008) clarified the role of early exposure in the development of preferences for the faces of conspecifics. He compared looking time for own and other species' faces in adult and juvenile Japanese monkeys (Macaca fuscata). Adult macaques raised in a monkey colony preferred 
to look at photographs of their conspecifics rather than at human faces or objects. Juvenile macaques reared without exposure to any faces showed a general preference for faces when paired with objects but no preference for monkey faces over human faces when they were paired. Following the period of face deprivation, the monkeys were then exposed to either real monkey or human faces. Within just 1 month they showed a visual preference for the type of faces they were exposed to. In the same study, Sugita also tested the monkeys' recognition/discrimination ability with a visual paired comparison (VPC) task whereby the visual preference test was preceded by a familiarization phase during which a human or monkey face was repeatedly presented. In the preference test shortly afterwards a new face from the same category was paired with the familiar face. Control monkeys looked longer at the novel faces only for the monkeys' faces but deprived monkeys looked longer at the novel faces for the category of faces they interacted with at the end of the deprivation period, either monkey or human.

Clearly, the viewing preference observed in Sugita's deprived monkeys was led by the exposure they had had. Further work has shown that experience with conspecific faces can also lead to changes in face-scanning behavior in both humans and monkeys. Like humans, rhesus monkeys use different strategies when scanning rhesus macaques and human faces, with monkey faces being processed more holistically (Dahl et al. 2007, 2009; Gothard et al. 2004, 2009). More specifically, these studies have shown that the spatial distribution of eye fixations is modulated by conspecificity, the eyeregion of conspecific faces receiving more attention than other face parts. Familiarity, defined as being an in-group member, and direct versus averted gaze also influenced single face scanning in macaques (Leonard et al. 2012). Again, the eyes of familiar individuals received more attention than the eyes of unfamiliar conspecifics. Preferential gaze towards the eye region of conspecific faces has also been found in chimpanzees (Hirata et al. 2010).

Although expertise with conspecific faces is associated with specific face scanning pattern, its influence on viewing time preferences is less clear. The results from Dahl et al. (2009) on rhesus macaques and from Sugita (2008) on Japanese monkeys suggest that conspecificity drives viewing preference when human and monkey faces are paired. However, recent results from Mahajan et al. (2011), showing longer looking time towards the faces of out-group members compared to in-group members, indicate that social categories such as group-membership can further explain differences in viewing time between faces from the same species. In the present research we proposed to examine how face category influences relative viewing preferences in both human and non-human primates. To address this issue, we conducted two experiments in which we presented Caucasian adult and rhesus macaque observers with pairs of faces, each from a different face category, including Caucasian and Asian humans, chimpanzees (Pan troglodytes), Barbary macaques (Macaca sylvanus) and rhesus macaques (Macaca mulatta). The rhesus macaques were socially housed indoors and daily exposed to conspecifics as well as masked and unmasked Chinese caretakers. In Experiment 1 we focused on the influence of novel face categories on viewing preferences, and built six different sets of face pairs using Asian and Caucasian faces as well as chimpanzee and Barbary macaque faces (Asian/Caucasian, Asian/chimpanzee, Asian/Barbary macaque, Caucasian/chimpanzee, Caucasian/Barbary macaque, and chimpanzee/Barbary macaque). In Experiment 2, we introduced conspecific faces and built four new sets to study the influence of conspecificity (Asian/rhesus macaque, Caucasian/rhesus macaque, chimpanzee/rhesus macaque, Barbary macaque/rhesus macaque). 
Following the results from Dahl et al. (2009) on rhesus macaques, and those from Sugita (2008) on Japanese monkeys, we expect longer looking time for the rhesus macaque faces when they are paired with human faces. If viewing preference in rhesus monkey is driven by conspecificity we could further predict that rhesus faces should be looked longer than the face of the other non-human primates. Alternatively, if novelty matters viewing time towards the unfamiliar faces (Barbary macaques, chimpanzees and, Caucasian humans) should be greater than toward Asian faces. If Caucasian faces and Asian faces attract similar amount of looking time it might indicate that both face types are categorized similarly. For our Caucasian human participants, novelty should increase their viewing time for rhesus macaques, chimpanzees and Asian faces when paired with Caucasian faces. Face categorization in humans is furthermore complicated by the existence of an "other-race effect", participants being better at recognizing own- than other-race faces (Michel et al. 2006; Ge et al. 2009). However, no increased visual attention towards own-race faces has been documented in human adults. In fact, familiarity would predict a preference for Caucasian faces which could be balanced by the novelty of the Asian faces, ending in a null preference.

\section{Materials and methods}

\section{Participants}

\section{Humans}

Twenty-three Caucasian adults participated in Experiment 1 (24-42 years, 15 women) and 18 other Caucasian adults participated in Experiment 2 (22-28 years, 10 women). They had no specific history of being familiar with non-human primates although they had all seen monkey faces in documentaries or books. Participation was rewarded by credits for an experimental Psychology course. The experiment on human subjects was conducted in the Psychology and NeuroCognition laboratory in Grenoble. All participants gave informed verbal consent and a local ethics committee approved the study.

\section{Monkeys}

Five male adult rhesus macaques ( $M$. mulatta, 5-9 kg, 5-9 years) participated in Experiments 1 and 2. Four out of the five monkeys participating in Experiment 1 also participated in Experiment 2 together with a new male adult. The interval between the two experiments was 6 months. All the monkeys were born in captivity and socially housed indoors. They grew up in large social groups and were exposed to masked and unmasked Chinese caretakers and to their conspecifics on a daily basis in Beijing (China). The monkeys had very limited experience with Caucasian faces although they could have occasionally seen Caucasian visitors. The monkeys had a limited experience of viewing photographs of human and monkey faces ( $<1$ week for each tested monkey) and were unfamiliar with the monkey and human faces used as stimuli. All monkeys were mid-ranked individuals in the hierarchy of the colony. The detailed experimental setup has been described in McFarland et al. (2013). Briefly, before the recording, a head restraint was implanted under aseptic conditions and monkeys were trained to fixate a small fixation point on a computer screen for a couple of seconds in exchange for juice reward. The animal experiments were conducted at Beijing Normal University, with all procedures in compliance with the US National Institutes of Health Guide for the Care and Use of Laboratory Animals, and approved by the Institutional Animal Care and Use Committee of Beijing Normal University. 


\section{Stimuli}

We used the faces of 16 Asians (8 women), 16 Caucasians ( 8 women), 8 Chimpanzees (P. troglodytes), 8 Barbary macaques (M. sylvanus) and 20 rhesus macaques faces to build the sets of face pairs used in Experiments 1 and 2. The photographs of the non-human primate faces were collected from different sources and the sexes were unknown. Faces were based on full frontal photographs with eyes open, direct frontal gaze, and neutral (mouth-closed) expression. All the faces used were unfamiliar to the human and macaque participants. The faces were normalized for luminance and the contours were masked by an oval frame ( $460 \times 520$ pixels). The background of the image $(1,024 \times 768$ pixels $)$ was filled with $30 \mathrm{~cd} / \mathrm{m}^{2}$ gray. The two faces were separated by 100 pixels (see Figs. $\underline{1}, \underline{2}$ for examples). 
example face pairs
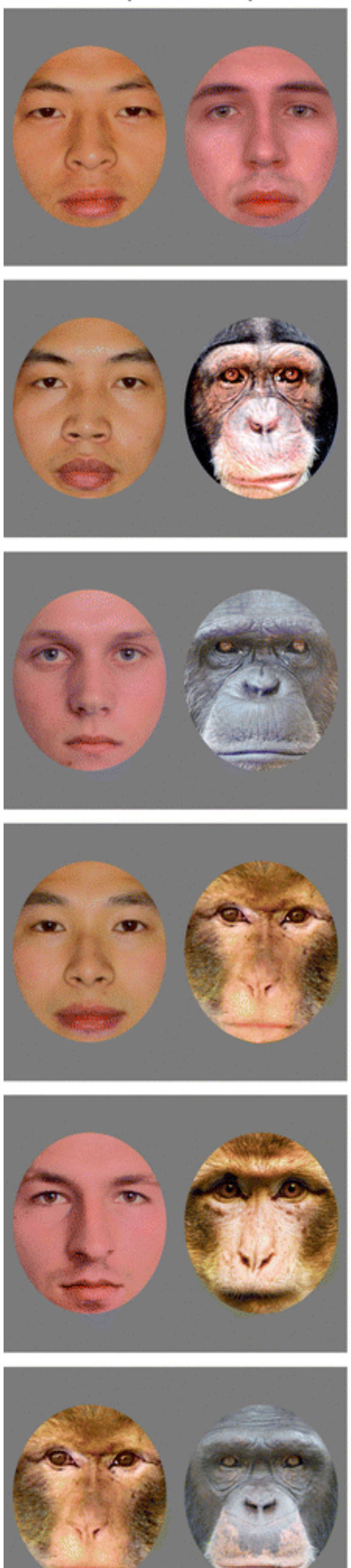

human

rhesus ma
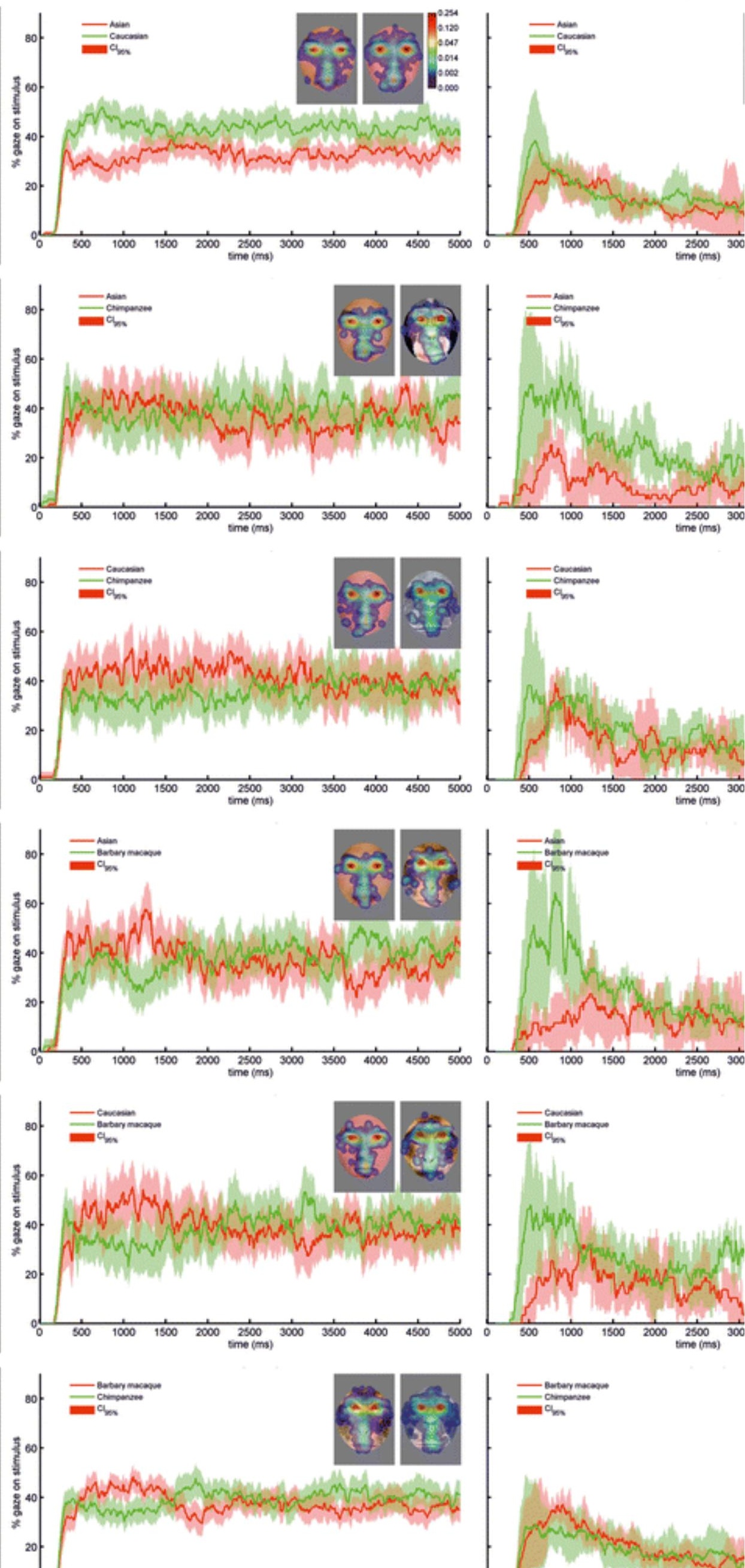
Fig. 1

Example face pairs and time course of viewing preferences for human and rhesus macaque participants in Experiment 1. The color ranges in heat maps were aligned to the maximum values found over all conditions and experiments 
example face pairs
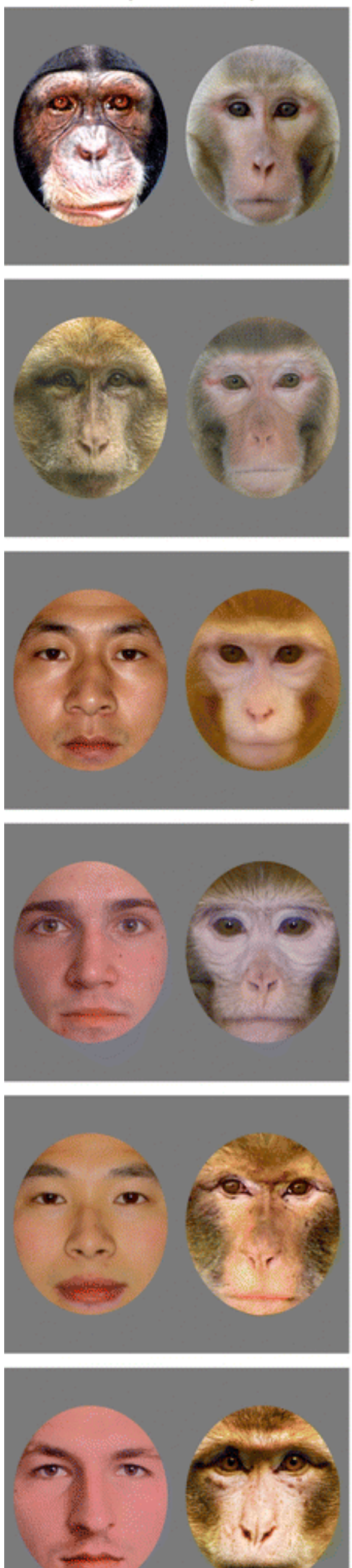

human
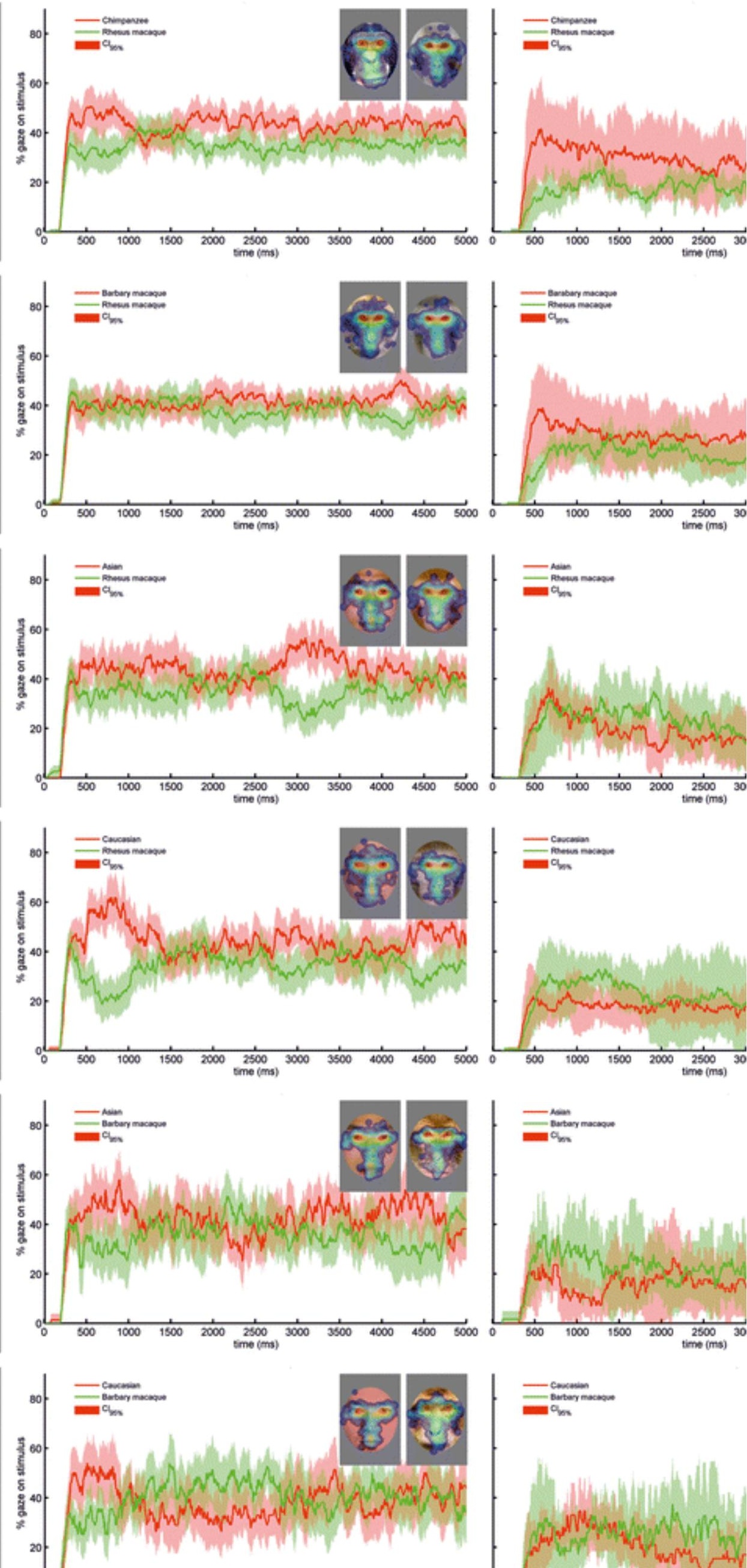
Fig. 2

Example face pairs and time course of viewing preferences for human and rhesus macaque participants in Experiment 2. The color ranges in heat maps were aligned to the maximum values found over all conditions and experiments

The stimuli used in Experiment 1 were grouped into six sets (or conditions, see Table $\underline{1}$ ) designed to test viewing preferences between two categories of faces. The 24 images/face-pairs were then duplicated and inverted to counterbalance the left-right position of the different face categories. The final experimental set comprised 48 images. We also used a set of 12 images showing two identical human faces to assess the effect of position on the screen. The analysis of viewing time on these control images showed no main effect of position and is not reported here.

Table 1

Number of stimuli per conditions

\begin{tabular}{|l|l|l|l|}
\hline Experiment 1 & & Experiment 2 \\
\hline Condition & n & Condition & n \\
\hline Asian-Caucasian & 8 & Chimpanzee-rhesus macaque & 8 \\
\hline Asian-Barbary macaque & 2 & Barbary macaque rhesus macaque & 8 \\
\hline Caucasian-Barbary macaque & 2 & Asian-rhesus macaque & 4 \\
\hline Asian-chimpanzee & 2 & Caucasian-rhesus macaque & 4 \\
\hline Caucasian-chimpanzee & 2 & Asian-Barbary macaque & 2 \\
\hline Chimpanzee-Barbary macaque & 8 & Caucasian-Barbary macaque & 2 \\
\hline
\end{tabular}

Each stimulus was duplicated and rotated to counterbalance the faces positions. The final sets comprise $n * 2$ stimulus images

The stimuli used in Experiment 2 were grouped into four sets designed to test changes in visual attention when conspecific faces were included (see Table 1 ). In order to check for the stability over time of the results found in Experiment 1 we also included the Asian-Barbary macaque and Caucasian-Barbary macaque sets of Experiment 1 . The 28 images/face-pairs were also duplicated and inverted to counterbalance the position of the different face category. The final experimental set comprised 56 images. Because we had fewer faces than needed to build the whole set of stimuli we reused some of the monkey faces when it was necessary.

\section{Visual preference task}

During the experiments, the monkeys were seated in a primate chair with their head restrained. Humans were also seated and head movements were limited with a chin rest. Both group of subjects 
viewed the display binocularly. Stimuli were displayed on a gamma-corrected colour monitor (liyama Vision Master Pro 514, $40 \times 30 \mathrm{~cm}$ ) with a resolution of 1,024 × 768 pixels and frame rate of $100 \mathrm{~Hz}$. A standard five-points calibration procedure for mapping gaze position to screen position was conducted before the beginning of each experimental session.

For rhesus monkeys, a trial was started with a fixation point displayed on the centre of the monitor. If the monkeys maintained fixation for $1 \mathrm{~s}$, the fixation point disappeared and one of the image was presented for $10 \mathrm{~s}$ (Experiment 1) or $5 \mathrm{~s}$ (Experiment 2). During the free-viewing presentation, the monkeys passively viewed the images. The inter-trial interval was $1 \mathrm{~s}$ within which the monkeys received a juice reward without any specific task requirement related to the stimuli. We ran three testing sessions, separated by 2-3 days, for each experiment. On each session the 48 stimuli of Experiment 1 or the 56 stimuli of Experiment 2 were shown in random order. Experiment 2 was run 6 months after the first experiment.

For humans, a trial was started with a fixation point displayed on the centre of the monitor. The experimenter triggered the image presentation manually after ensuring that the participant was fixated on the fixation point. A drift correction was performed every 5 trials to realign gaze and screen spaces and correct eventual involuntary head displacements. The stimulus was presented for $5 \mathrm{~s}$ and the instructions emphasized free viewing of the images. A testing session comprised all the stimuli in random order. Only one session per experiment was run for human viewers.

\section{Eye-tracking measurements and data processing}

During the testing, horizontal and vertical eye positions from monkey and human participants were measured by EyeLink 1000 (SR Research Ltd., Mississauga, Ontario, Canada). For technical reasons, sampling frequency and viewing distance were $500 \mathrm{~Hz}$ and $100 \mathrm{~cm}$ for monkeys and $250 \mathrm{~Hz}$ and 60 $\mathrm{cm}$ for humans. All calculations for the identification of fixations were done in degrees of visual angle. For simplicity, and because monkeys barely looked at the stimulus when exposure exceeded a few seconds, only the first 5 s of monkeys' data were used in Experiment 1, to match the presentation time used in humans. The parsing of the eye data into saccade and fixation was done according to the method developed by Engbert and Kliegl (2003). The fixations within each trial were classified according to their locations (on first face, on second face, or elsewhere on the screen). Only trials where participants gazed at one of the two faces and with the initial gaze on the fixation point were included in the dataset.

For the main statistical analysis, the total looking times on each face were calculated for each participant and for each trial in a session. The results from the different trials in each condition were averaged for each participant leading to a single estimate of viewing time on each face category per session, condition, and participant. Averaging over the trials was required because of the different numbers of stimuli in each set (see Table 1 ). The goal of the main analysis was to test whether the looking times on the two face categories within a set of images (or condition) were different. To do so we used a mixed-linear model including condition, session and face category as fixed-effect factors and the subject as a random effect factor. The model could be written as follow: $L T=\beta_{1} C+$ $\beta_{2} S+\beta_{3} C F+\alpha_{s}+\varepsilon$ where LT stands for looking time, $C$ for condition (see Table 1 for levels of this factor), $S$ for session $(1,2,3)$, and $C F$ for the interaction of the condition with the face category. Face category was coded as follows. We took the first face category in a set (e.g., Asian in the AsianCaucasian set) and gave it the value -0.5. The other face (e.g., Caucasian) was given the value 0.5. 
The values of $\beta_{1}$, reflected the mean time spent on the faces in each condition. The values of $\beta_{2}$ reflected the changes in average looking time on faces in sessions 2 and 3. The term $\beta_{2} S$ was removed for the analysis of human data because human participants did only one session. Of particular interest here are the values for the coefficients $\beta_{3}$ which directly represent the differences in looking time (in $\mathrm{ms}$ ) between the two face categories in each condition. A significant negative value indicates that the first face category in a set was looked at more than the second face category (i.e., Asian > Caucasian). A significant positive value indicates the opposite (i.e., Asian < Caucasian). Finally, the coefficients $\alpha_{s}$ (one per subject) represented the subjects' random effects. A mixed-effect model allowed us to control for idiosyncrasies in average looking behavior, especially in the monkey group. Other models, used to test more specific hypotheses, are given in the result section. Stimulus presentation and data processing were done with Matlab ${ }^{\circ}$ and the EyeLink Toolbox. Statistical analyses were done with the nlme package in $\mathrm{R}$ ( $\mathrm{R}$ Development Core Team 2008). Additional details on the implementation of the statistical model can be found in the Supplemental Data available online.

\section{Results}

\section{Experiment 1}

The mean looking time on each of two simultaneously presented faces and on the first and second face category for each condition are given in Tables $\underline{2}$ and $\underline{3}$ for rhesus macaques and humans respectively.

Table 2

Results for rhesus macaques

\begin{tabular}{|c|c|c|c|c|c|}
\hline Condition & Mean viewing time per faces & $\beta_{1} C^{a}$ & Face $=1$ & Face $=2$ & $2-1$ \\
\hline \multicolumn{6}{|l|}{ Experiment 1} \\
\hline Asian-Caucasian & 643 & 0 & 583 & 704 & 121 \\
\hline Asian-chimpanzee & 685 & 42 & 420 & 950 & $530 * * *$ \\
\hline Caucasian-chimpanzee & 638 & -5 & 544 & 734 & 190 \\
\hline Asian-Barbary macaque & 733 & 90 & 493 & 973 & $480 * * *$ \\
\hline Caucasian-Barbary macaque & 985 & $342 * *$ & 600 & 1,370 & $770 * * *$ \\
\hline Barbary macaque-chimpanzee & 785 & 142 & 789 & 781 & -8 \\
\hline \multicolumn{6}{|l|}{ Experiment 2} \\
\hline Chimpanzee-rhesus macaque & 1,112 & 0 & 1,358 & 866 & $-491 * * *$ \\
\hline
\end{tabular}




\begin{tabular}{|l|l|l|l|l|l|l|}
\hline Condition & Mean viewing time per faces & $\boldsymbol{\beta}_{1} \mathbf{C}^{\mathbf{a}}$ & Face = 1 & Face = 2 & $\mathbf{2 - 1}$ \\
\hline Barbary macaque-rhesus macaque & 1,059 & -53 & 1,224 & 894 & $-330^{*}$ \\
\hline Asian-rhesus macaque & 936 & -176 & 811 & 1,061 & 250 \\
\hline Caucasian-rhesus macaque & 941 & -171 & 815 & 1,068 & 253 \\
\hline Asian-Barbary macaque & 922 & -190 & 674 & 1,170 & $496^{* * *}$ \\
\hline Caucasian-Barbary macaque & 1,057 & -55 & 977 & 1,138 & 161 \\
\hline
\end{tabular}

Mean viewing time per faces in experiments 1 and 2 and mean viewing times for each face category. Values are given in milliseconds. Changes in mean viewing time per faces were assessed using a linear model with intercept: $L T=\beta_{0}+\beta_{1} C^{a}+\varepsilon$

$* \mathrm{P}<0.05 ; * * \mathrm{P}<0.01 ; * * * \mathrm{P}<0.001$

Table 3

Results for humans

\begin{tabular}{|c|c|c|c|c|c|}
\hline Condition & Mean viewing time per faces & $\beta_{1} C^{a}$ & Face $=1$ & Face $=2$ & $2-1$ \\
\hline \multicolumn{6}{|l|}{ Experiment 1} \\
\hline Asian-Caucasian & 1,816 & 0 & 1,553 & 2,079 & $526 * * *$ \\
\hline Asian-chimpanzee & 1,801 & -15 & 1,749 & 1,854 & 104 \\
\hline Caucasian-chimpanzee & 1,837 & 21 & 1,981 & 1,693 & $-288^{*}$ \\
\hline Asian-Barbary macaque & 1,811 & -5 & 1,796 & 1,826 & 29 \\
\hline Caucasian-Barbary macaque & 1,859 & 43 & 1,880 & 1,837 & -43 \\
\hline Barbary macaque-chimpanzee & 1,817 & 1 & 1,778 & 1,857 & 79 \\
\hline \multicolumn{6}{|l|}{ Experiment 2} \\
\hline Chimpanzee-rhesus macaque & 1,879 & 0 & 2,078 & 1,680 & $-398 * * *$ \\
\hline Barbary macaque-rhesus macaque & 1,898 & 19 & 1,964 & 1,831 & -133 \\
\hline Asian-rhesus macaque & 1,883 & 4 & 2,082 & 1,685 & $-397 * * *$ \\
\hline
\end{tabular}




\begin{tabular}{|l|l|l|l|l|l|l|}
\hline Condition & Mean viewing time per faces & $\boldsymbol{\beta}_{1} \mathbf{C}^{\mathbf{a}}$ & Face $=\mathbf{1}$ & Face $=\mathbf{2}$ & $\mathbf{2}-\mathbf{1}$ \\
\hline Caucasian-rhesus macaque & 1,878 & -1 & 2,128 & 1,629 & $-499 * * *$ \\
\hline Asian-Barbary macaque & 1,869 & -10 & 2,027 & 1,712 & $-314^{* *}$ \\
\hline Caucasian-Barbary macaque & 1,880 & 1 & 1,835 & 1,924 & 89 \\
\hline
\end{tabular}

Mean viewing time per faces in experiments 1 and 2 and mean viewing time for each face category. Values are given in milliseconds. Changes in mean viewing time per faces were assessed using a linear model with intercept: $L T=\beta_{0}+\beta_{1} C^{a}+\varepsilon$

$* \mathrm{P}<0.05 ; * * \mathrm{P}<0.01 ; * * * \mathrm{P}<0.001$

\section{Rhesus macaques}

As shown in Table 2 , the mean looking time was significantly increased in the Caucasian-Barbary macaques condition $\left(\mathrm{t}_{174}=2.86, \mathrm{P}<0.01\right)$ with respect to the Asian-Caucasian condition. The differences between the mean viewing time on face category 1 and 2 were also given in Table $\underline{2}$. Rhesus macaques spent more time looking at the chimpanzee faces in the Asian-chimpanzee condition $\left(\mathrm{t}_{162}=3.73, \mathrm{P}<0.001\right)$, at the Barbary macaque faces in the Asian-Barbary macaque $\left(\mathrm{t}_{162}=\right.$ $3.38, \mathrm{P}<0.001)$ and Caucasian-Barbary macaque $\left(\mathrm{t}_{162}=5.42, \mathrm{P}<0.001\right)$ conditions. The mean viewing time on testing session 2 and 3 were shorter than in session 1 but these differences were not significant (session $2=-112 \mathrm{~ms}, \mathrm{t}_{162}=1.49$, NS; session $3=-91 \mathrm{~ms}, \mathrm{t}_{162}=1.21$, NS). The random effects for the five monkeys were the following: monkey $C=-47 \mathrm{~ms}$, monkey $\mathrm{D}=224 \mathrm{~ms}$, monkey $\mathrm{G}$ $=0 \mathrm{~ms}$, monkey $\mathrm{I}=-91 \mathrm{~ms}$, and monkey $\mathrm{J}=-85 \mathrm{~ms}$. Monkeys $\mathrm{D}$ and I were respectively the most and the least interested in the stimuli. Finally, we grouped the data in the Asian-chimpanzee and Caucasian-chimpanzee conditions and in the Asian-Barbary macaque and Caucasian-Barbary macaque conditions to form a human-chimpanzee and a human-Barbary macaque condition. The results indicated that the rhesus macaques spent more time looking at the non-human primate faces than at human faces (human-chimpanzee, $\mathrm{t}_{106}=3.16, \mathrm{P}<0.01$; human-Barbary macaque, $\mathrm{t}_{106}=$ $5.99, \mathrm{P}<0.001)$.

\section{Humans}

As shown in Table $\underline{3}$, the mean looking time was about three times longer in human viewers than in the monkey viewers and no differences between the Asian-Caucasian condition and the other conditions was found in the human group. The differences in viewing time on face category 1 and 2 indicated that Caucasian humans spent more time looking at the Caucasian faces in Asian-Caucasian $\left(\mathrm{t}_{242}=4.1, \mathrm{P}<0.001\right)$ and Caucasian-chimpanzee $\left(\mathrm{t}_{242}=-2.24, \mathrm{P}<0.05\right)$ conditions. No differences in looking times between two faces were found for the other conditions.

\section{Experiment 2}

The results of Experiment 1 indicated that rhesus macaques' attention was captured by nonconspecific monkey faces, which were looked at longer than the human faces. Are these novel faces still preferred when presented together with unfamiliar conspecific faces? Experiment 2 was 
designed to examine this question, and the mean looking times for each of two simultaneously presented faces and on the first and second face category for each condition were given in Tables $\underline{2}$ and $\underline{3}$ for rhesus macaques and humans respectively.

\section{Rhesus macaques}

As shown in Table 2 , rhesus macaques spent more time looking at the chimpanzee faces in the chimpanzee-rhesus macaque condition $\left(\mathrm{t}_{162}=-3.48, \mathrm{P}<0.001\right)$, and at the Barbary macaque faces in the Barbary macaque-rhesus macaque $\left(\mathrm{t}_{162}=-2.33, \mathrm{P}<0.05\right)$ and Asian-Barbary macaque $\left(\mathrm{t}_{162}=\right.$ $3.51, \mathrm{P}<0.001)$ conditions. The results from the Asian-rhesus macaque and Caucasian-rhesus macaque conditions indicated a trend towards a longer looking time for the rhesus macaque faces but these differences were not significant at the $5 \%$ threshold (Asian-rhesus macaque, $t_{162}=1.77$, NS; Caucasian-rhesus macaque, $\mathrm{t}_{162}=1.79$, NS). As in Experiment 1, we grouped the data in the Asian-rhesus macaque and Caucasian-rhesus macaque conditions and in the Asian-Barbary macaque and Caucasian-Barbary macaque conditions to form a human-rhesus macaque (humanrhesus macaque) condition and a human-Barbary macaque (human-Barbary macaque) condition. In both conditions, the rhesus macaques spent more time looking at the unfamiliar faces of rhesus and Barbary macaques than at human faces (human-rhesus macaque, $t_{108}=2.08, P<0.05$; humanBarbary macaque, $\left.\mathrm{t}_{108}=2.59, \mathrm{P}<0.05\right)$.

The mean viewing time in testing session 2 and 3 was shorter than in session 1 and this difference was significant in session 2 ( $\left.M=-264 \mathrm{~ms}, \mathrm{t}_{162}=-3.74, \mathrm{P}<0.001\right)$ but not in session $3\left(\mathrm{M}=-122, \mathrm{t}_{162}\right.$ $=-1.72, \mathrm{NS})$. The random effects for the five monkeys were as follows: monkey $\mathrm{H}=798 \mathrm{~ms}$, monkey $\mathrm{D}=-47 \mathrm{~ms}$, monkey $\mathrm{G}=15 \mathrm{~ms}$, monkey $\mathrm{I}=-163 \mathrm{~ms}$, monkey $\mathrm{J}=-604 \mathrm{~ms}$ ). Monkeys $\mathrm{H}$ and $\mathrm{J}$ were the most and the least interested in the stimuli. In this second experiment we re-used the Caucasian-Barbary macaque and Asian-Barbary macaque conditions of Experiment 1 to test the repeatability of the results. We found a significant preference for Barbary macaques over Asian faces in both experiments. The preference for Barbary macaque over Caucasian faces was in the same direction as in Experiment 1 but the difference was not significant. It is possible that repeated exposure to the stimuli for four out of the five monkeys who participated in both experiments influenced the results in this condition.

\section{Humans}

As shown in Table $\underline{3}$, the mean looking time on faces was again longer in the human group than in the monkey group, and no differences between the chimpanzee-rhesus macaque condition and the other conditions was found. Furthermore, Caucasian humans spent more time looking at the chimpanzee faces in the chimpanzee-rhesus macaque condition ( $\left.\mathrm{t}_{198}=4.04, \mathrm{P}<0.001\right)$, at the Asian and Caucasian faces in the Asian-rhesus macaque $\left(\mathrm{t}_{198}=-4.04, \mathrm{P}<0.001\right)$ and Caucasian-rhesus macaque $\left(t_{198}=-5.07, P<0.001\right)$ conditions. The human participants also looked longer at the Asian faces in the Asian-Barbary macaque conditions.

\section{Post-hoc analysis}

\section{Contrasting viewing time on Asian and Caucasian faces}

In Experiments 1 and 2, the rhesus macaques were exposed to Asian and Caucasian faces both when they were paired together and when they were paired with chimpanzee, Barbary macaque and 
rhesus macaque faces. From the mean viewing time per face column in Table $\underline{2}$ it appears that in all cases the Asian faces were looked at for less time than the Caucasian faces. However, contrasting the time spent looking at the Asian faces in the Asian-Caucasian, Asian-Barbary macaque, Asianchimpanzee and Asian-rhesus macaque conditions with the time spent looking at Caucasian faces in the Asian-Caucasian, Caucasian-chimpanzee, Caucasian-Barbary macaque and Caucasian-rhesus macaque conditions showed that this difference was not significant (Asian: $M=535 \mathrm{~ms}$, Caucasian: $\left.M=636 \mathrm{~ms}, \mathrm{t}_{24}=-1.93, \mathrm{NS}\right)$.

\section{Time course of viewing preferences and spatial distribution of on-faces fixations}

The eye-tracking data collected in Experiments 1 and 2 could potentially lead to a detailed analysis of the face scanning strategies used by the humans and rhesus macaques. However, face scanning strategy was not a central issue for the analysis of viewing preferences in our experiments and we focused on the analysis of relative viewing time. In this section, mostly descriptive, we provide more details on the time course of viewing preference and the spatial distribution of gaze over two simultaneously presented faces. For more information on the face scanning strategies used by human and monkeys, the readers should refer to Dahl et al. (2009) and Gothard et al. (2004, 2009).

In addition to the measurements of total looking time on each of the two faces shown in a trial, we also calculated the proportion of gaze on each face as a function of time. Practically, the time course of viewing behavior was represented, for each measurement sample with ones when gaze was on the face and zero otherwise. These vectors were used to produce the raster plots shown in the Supplemental Data available online. For each participant and condition, these binary vectors ( 2 per trials, one per face category) were first averaged over all the faces from the same category in a condition taking into account the position of the face from each category (face category 1 on the left or on the right, face category 2 on the right or on the left). Then we averaged the vector from the two presentation sides (face category 1 on the left and face category 1 on the right). This procedure ensured that each presentation side would have the same weight in the time course estimation. Finally, the average over the subjects was used to represent the time course and the $95 \%$ confidence intervals of on-face gaze position in each condition shown in Figs. $\underline{1}$ and $\underline{2}$.

The most noticeable feature of the time course of viewing preference for the rhesus macaques participating in Experiment 1 was the large peak towards the novel non-human primate species in the Asian-chimpanzee, Asian-Barbary macaque, and Caucasian-Barbary macaque conditions. These peaks indicates that following image presentation the five rhesus macaques involved in Experiment 1 behaved in the same way, making frequent looks towards the chimpanzee and Barbary macaque faces. In humans, the viewing preference towards Caucasian faces, when they were paired with Asian faces, could be seen from the first look and was maintained throughout the stimulus presentation time.

In Experiment 2 a preference for the chimpanzee and Barbary macaque faces could be seen from trial onset when these were paired with rhesus macaque faces but the strong responses observed in Experiment 1 were somewhat attenuated. This difference from Experiment 1 could stem from the previous experience that four out of the five rhesus macaque participating in Experiment 2 had with chimpanzee and Barbary macaque faces. Nevertheless, in these conditions significant preferences for the faces of monkeys from other species were found as shown in Table $\underline{2}$. The viewing preferences in human participants changed over time in a way that is not easy to summarize. 
Sometimes, as in the Caucasian-rhesus macaque, a strong initial bias towards human face could be observed, but in most of the other conditions preferences varied in an unpredictable manner.

We also represented the spatial distribution of viewing time over the face area for the human and the monkey group using heat maps (see Figs. $\underline{1}, \underline{2}$ and the Supplemental Data available online for enlarged version). The total duration of the fixations on each face, in milliseconds, were cumulated in grids on a pixel by pixel basis. The grids were smoothed with a bi-dimensional Gaussian with a width of $2^{\circ}$ and a standard deviation of $0.5^{\circ}$. The smoothed values in the grids were then divided by the number of valid trials in the group. Thus, the sum of all density values in a grid was equal to the group's average viewing time at each face in a given condition. The grids were overlaid on the first face pair in the image set of each testing condition. Because the distributions of the densities were not Gaussian, but rather distributed according to an exponential decay function, we used a cubic root transform before mapping densities with color levels (see the color bar levels in the top plot of Fig. 1). The maximum value in the color map for the human and rhesus macaque participants was fixed at the maximum smoothed value found over the two experiments for the human and rhesus macaque datasets.

The data represented in Figs. $\underline{1}$ and $\underline{2}$ are mainly descriptive. Heat map subtraction, often used to contrast exploration of one stimulus in different group cannot be used here because the features (eyes, nose, and mouth) in the faces from each category had different locations and shapes. However, the subtraction of the human and monkey maps for the same stimulus always indicated that the centre of the eyes, in any of the face used, was looked at more by humans than by monkeys. This peculiarity of the human observers was evident in Figs. $\underline{1}$ and $\underline{2}$.

\section{Discussion}

In two experiments, we presented pairs of faces from two human races and three non-human primate species to both humans and rhesus macaques viewers and compared the mean viewing time for each face category. The results showed that the human participants looked more at human faces from their own-race group (Caucasian) than at face from another race group (Asian). In addition, Caucasians spent more time looking at Caucasians than at chimpanzees, at chimpanzees than at rhesus macaques, at humans (Caucasian or Asian) than at rhesus macaques, and at Asians than at Barbary macaques. The rhesus macaques involved in this study looked more at non-human primate faces than at human faces. When faces from different species of non-human primate were presented together with conspecifics' faces the rhesus macaques looked longer at the other species' faces (chimpanzees or Barbary macaques).

Our findings of an own-race bias in the human group can be interpreted in light of the "other-race" effect (Michel et al. 2006). Humans are better at recognizing own- than other-race faces in old-new tasks and are faster at detecting other-race faces in categorization tasks (Ge et al. 2009). These two sides of the other-race effect are related to exposure and can be much reduced by perceptual training (Heron-Delaney et al. 2011) or by facial expression of positive emotions (Johnson and Fredrickson 2005). Despite considerable work on the other-race effects in categorization and recognition tasks, increased visual attention towards own-race faces in visual preference tasks has never been documented in human adults. Our results indicate that the attentional bias towards own-race faces, which can be observed as early as 3 months of age in human infants (Kelly et al. 2005), carries on in adulthood. 
An attentional bias towards own-race faces in our Caucasian participants was also observed when the Caucasians were paired with chimpanzees in Experiment 1 or with rhesus macaques in Experiment 2. However, in both experiments the own-race bias was not found when Caucasians were paired with Barbary macaques. This discrepancy suggests that the own-race bias was not the only factor influencing visual attention in the testing condition and that other factors related to the faces of the Barbary macaques balanced the bias towards own-race faces.

A modulation of viewing preference in relation to the pairing of the face type was also observed in the rhesus macaques. In Experiment 1, with the exception of the Caucasian-chimpanzee condition, the faces of other non-human primate species were always looked longer than the human faces. The overall preference for monkey faces, found when the results from both human races were grouped, confirmed this conclusion. In Experiment 2 we also found that non-human primate faces (including conspecifics' faces) were looked at longer than human faces when Asian and Caucasian faces were grouped. Longer looking time toward conspecifics was expected from Sugita's results (2008). In the present study, the repeated findings of shorter looking times toward Asians, and more generally, human faces can also be interpreted as a submissive behavior, given gaze aversion generally indicates anxiety and submissiveness (Deaner et al. 2005). The rhesus macaques involved in this study were all familiar with Asian faces. They were at least partially raised by Asians, and nurtured and trained by Asians who most probably had a dominant status. Caucasian faces were not looked at longer than Asian faces suggesting that the Caucasian faces were assimilated into the broader category of human faces and were probably considered as members of a dominant group by the rhesus macaques involved in this experiment.

The bias towards non-human primate faces from other species was also found in Experiment 2, when non-conspecific faces were paired with unfamiliar conspecifics. This finding may seem at odds with the results from Fujita (1987) showing that macaques from different species prefer to look at conspecific faces rather than faces of other macaque species. However, in his experiment, Fujita (1987) used a self-controlled single face presentation task in which monkeys had to press a lever to see either conspecifics or other macaques' faces. Our visual preference task is different and more closely matches the setup used by Mahajan et al. (2011). In the first experiment of this latter study, the authors presented two rhesus macaques faces simultaneously in a visual preference setting and found longer looking time toward the faces of out-group conspecifics than towards in-group conspecifics. Here we found longer looking time towards other non-human primate species' faces that can similarly be accounted for by social categorization. Chimpanzees and Barbary macaques, which were relatively novel to our rhesus macaques, elicited more vigilance than conspecifics or humans. This result extends those from Mahajan et al. (2011) by showing that social categorization (i.e., as an out-group member) and the associated threat detection/perception are not restricted to conspecific faces but can guide visual attention towards novel species' faces. Returning to the results of Fujita (1987), we can assume that when given the choice by actively controlling the appearance of the faces, rhesus macaques had greater motivation to see conspecifics than potentially threatening novel species.

The pattern of preferences found in our two experiments cannot be accounted for by familiarity, which would predicts that Asians and rhesus macaques' faces would be looked longer than Caucasians, chimpanzees or Barbary macaques' faces, nor by conspecificity, which would have led to increased attention towards rhesus macaques' faces. Our interpretation of the results is that when 
conspecific faces were paired with human faces, both the dominant status of human faces and conspecificity of the rhesus macaque faces influenced viewing behavior towards own-species faces, even though these were not from familiar individuals. The same result was found by Dahl et al. (2009). However, according to the results of Mahajan et al. (2011), the unfamiliar conspecific faces would have been looked at longer if they had been paired with familiar rhesus macaques. Here, the rhesus faces elicited little attention compared to the faces from other monkey species. Indeed in Experiment 2, when rhesus faces were paired with faces from unfamiliar species, the out-group status of these individuals was particularly salient leading to increased attention towards these faces.

These results from the viewing preference task, showing that the visual attention allocated to a face is influenced by the combination of face types, illustrate an observation often made in categorization tasks. Faces can be assigned to multiple, partially overlapping, social categories that may competes and inhibit each other (Macrae et al. 1995; Higgins 1996). Specifically, a face can simultaneously be categorized as a human, a man, a famous person, an ethnic minority member, and so on, and the activation of a particular category can be influenced by task context. Situations where two faces are presented simultaneously, as in the visual preference paradigm, are thus likely to enhance categorical traits such as out-group status. Other recent results have shown that social roles in social interaction scenes influence the allocation of visual attention in rhesus macaques (McFarland et al. 2013). When presented with negative scenes, showing aggressive interaction between two rhesus macaques, rhesus macaques pay more attention toward the receiver (i.e., the victim of the aggression) than toward the giver (i.e., the aggressor). Interestingly, this pattern of result is reversed when they view negative scenes involving non-conspecific individuals (e.g., babbons and lions). In these cases, the giver receives more attention. Taken together these results suggest that rhesus macaques have role-sensitive, species- and context-dependent cognitive mechanisms for the regulation of social attention.

Social categorization of the novel species' faces as out-group members can give a coherent account for our monkey data, and suggest that novelty influences the viewing preferences by triggering behavioral responses related to social regulation. Novelty was not so influential in human observers who, overall, often preferred human faces and were particularly attracted by own-race faces only when those were paired with Asian faces. This reduced role of novel, out-group faces could be a distinctive feature of human cognition and indicate that for our human participants social regulation was not at stake here. However, although we can say that the chimpanzees and Barbary macaques' faces were truly novel for rhesus macaques the same is not true for our human participants who could have had many opportunities to see monkey and ape faces in zoos, movies and documentaries as well as Asian's faces. Future works using faces from monkey species that are not as popular as macaques and chimpanzees may help to shed light on these issues.

The results of this study, and those from Mahajan et al. (2011) and McFarland et al. (2013), have methodological implications for future researches using paired presentation. The type of comparison stimuli employed in facial recognition/preference tasks should be carefully chosen and controlled for. For example, if one is investigating species-specific effects, the pairing of the conspecific faces with human faces, out-group conspecifics or other non-human primate species' face will impact results. 


\section{Acknowledgments}

We thank two anonymous reviewers for their fruitful comments on an early version of the manuscript. This research was supported by National Natural Science Foundation of China Grant 31125014, Open Research Fund of the State Key Laboratory of Cognitive Neuroscience and Learning (China) and by a grant from the National Institutes of Health Grant R01 HD046526 (to O. P.).

Conflict of interest

The authors declare that they have no conflict of interest.

Ethical standard

In accordance with French standards for behavioral studies, the experiment was approved by the local ethics committee of the Psychology and NeuroCognition Lab (human participants). The animal experiments were conducted at Beijing Normal University, with all procedures in compliance with the US National Institutes of Health Guide for the Care and Use of Laboratory Animals, and approved by the Institutional Animal Care and Use Committee of Beijing Normal University.

\section{Electronic supplementary material}

Below is the link to the electronic supplementary material.

Supplementary material 1 (PDF $8807 \mathrm{~kb}$ )

References

Bornstein RF (1989) Exposure and affect: overview and meta-analysis of research. Psychol Bull 106:265-289. doi:10.1037/0033-2909.106.2.265CrossRef

Bruce V, Young AW (1998) In the eye of the beholder: the science of face perception. Oxford University Press, Oxford, ISBN: 0198524404

Dahl CD, Logothetis NK, Hoffman KL (2007) Individuation and holistic processing of faces in rhesus monkeys. Proc R Soc Lond B 274:2069-2076. doi:10.1098/rspb.2007.0477CrossRef

Dahl CD, Wallraven C, Bulthoff HH, Logothetis NK (2009) Humans and macaques employ similar faceprocessing strategies. Curr Biol 19:1-5. doi:10.1016/j.cub.2009.01.061.ECrossRef

Deaner RO, Khera AV Platt ML (2005) Monkeys pay per view: adaptive valuation of social images by rhesus macaques. Curr Biol 15: 543-548, ISSN:0960-9822

Di Giorgio E, Méary D, Pascalis O, Simion F (2013) The face perception system becomes species specific at three months: an eye-tracking study. Int J Behav Dev 37:95-99. doi:10.1177/ 0165025412465362 CrossRef

Engbert R, Kliegl R (2003) Microsaccade uncover the orientation of covert attention. Vis Res 43:1035-1045. doi:10.1016/S0042-6989(03)00084-1PubMedCrossRef

Enquist M, Arak A (1994) Symmetry, beauty and evolution. Nature 372:169-172. doi:10.1038/ 372169a0PubMedCrossRef 
Fantz RL (1964) Visual experience in infants: decreased attention to familiar patterns relative to novel ones. Sci New Ser 146:668-670

Fujita K (1987) Species recognition by five macaque monkeys. Primates 28:353-366. doi:10.1007/ BF02381018CrossRef

Ge L, Zhang H, Wang Z, Quinn PC, Pascalis O, Kelly D, Lee K (2009) Two faces of the other-race effect: recognition and categorisation of Caucasian and Chinese faces. Perception 38:1199-1210. doi:10. 1068/p6136PubMedCrossRef

Gothard KM, Erickson CA, Amaral DG (2004) How do rhesus monkeys (Macaca mulatta) scan faces in a visual paired comparison task? Anim Cogn 7:25-36. doi:10.1007/s10071-003-0179-

6PubMedCrossRef

Gothard KM, Brooks KN, Peterson MA (2009) Multiple perceptual strategies used by macaque monkeys for face recognition. Anim Cogn 12:155-167. doi:10.1007/s10071-008-0179-

$\underline{\text { 7PubMedCrossRef }}$

Heron-Delaney M, Anzures G, Herbert JS, Quinn PC, Slater AM, Tanaka JW, Pascalis O (2011) Perceptual training prevents the emergence of the other race effect during infancy. PLoS One 6:e19858. doi:10.1371/journal.pone.0019858PubMedCentralPubMedCrossRef

Higgins ET (1996) Knowledge activation: accessibility, applicability, and salience. In: Higgins ET, Kruglanski AW (eds) Social psychology: handbook of basic principles. Guilford Press, New York, pp 133-168, ISBN:10:1-57230-918-0

Hirata S, Fuwa K, Sugama K, Kusunoki K, Fujita S (2010) Facial perception of conspecifics: chimpanzees (Pan troglodytes) preferentially attend to proper orientation and open eyes. Anim Cogn 13:679-688. doi:10.1007/s10071-010-0316-yPubMedCrossRef

Johnson KJ, Fredrickson BL (2005) We all look the same to me: positive emotions eliminate the ownrace bias in face recognition. Psychol Sci 16:875-881. doi:10.1111/j.1467-9280.2005.01631. $\underline{\text { xpubMedCentralPubMedCrossRef }}$

Kelly DJ, Quinn PC, Slater AM, Lee K, Gibson A, Smith M, Pascalis O (2005) Three-month-olds, but not newborns, prefer own-race faces. Dev Sci 8:F31-F36. doi:10.1111/j.1467-7687.2005.0434a. xPubMedCentralPubMedCrossRef

Leinbach MD, Fagot BI (1993) Categorical habituation to male and female faces: gender schematic processing in infancy. Infant Behav Dev 16:317-332. doi:10.1016/j.bbr.2011.03.031CrossRef Leonard TK, Blumenthal G, Gothard KM, Hoffman KL (2012) How macaques view familiarity and gaze in conspecific faces. Behav Neurosci 126:781-791. doi:10.1037/a0030348PubMedCrossRef

Macrae CN, Bodenhausen GV, Milne AB (1995) The dissection of selection in person perception: inhibitory processes in social stereotyping. J Pers Soc Psychol 69:397-407. doi:10.1037/0022-3514. 69.3.397PubMedCrossRef 
Mahajan N, Martinez MA, Gutierrez NL, Diesendruck G, Banaji MR, Santos LR (2011) J Pers Soc Psychol 100: 387-405. doi: 10.1037/a0022459

McFarland R, Roebuck H, Yan Y, Majolo B, Li W, Guo K (2013) Social interactions through the eyes of macaques and humans. PLoS One 8:e56437. doi:10.1371/journal.pone.

0056437PubMedCentralPubMedCrossRef

Michel C, Caldara R, Rossion B (2006) Same-race faces are perceived more holistically than otherrace faces. Vis Cogn 14:55-73. doi:10.1080/13506280500158761CrossRef

Park J, Shimojo E, Shimojo S (2010) Roles of familiarity and novelty in visual preference judgments are segregated across object categories. Proc Natl Acad Sci 107:14552-14555. doi:10.1073/pnas. 1004374107PubMedCentralPubMedCrossRef

Parr LA (2011) The evolution of face processing in primates. Philos Trans R Soc B 366:1764-1777. doi:10.1098/rstb.2010.0358CrossRef

Pascalis O, Wirth S (2011) Recognizing the face of other species: What can a limited skill tell us about face processing? In: Calder A, Rhodes G, Johnson MH, Haxby JV (eds) The Oxford handbook of face perception. Oxford Library of Psychology, Oxford, pp 719-730, ISBN:978-0199559053

Quinn PC, Yahr J, Kuhn A, Slater AM, Pascalis O (2002) Representation of the gender of human faces by infants: a preference for female. Perception 31:1109-1122. doi:10.1068/p3331PubMedCrossRef

R Development Core Team (2008) R: a language and environment for statistical computing. Vienna, Austria. http://www.R-project.org

Rentschler I, Jüttner M, Unzicker A, Landis T (1999) Innate and learned components of human visual preference. Curr Biol 9:665-671. doi:10.1016/S0960-9822(99)80306-6PubMedCrossRef

Sugita Y (2008) Face perception in monkeys reared with no exposure to faces. Proc Natl Acad Sci USA 105:394-398. doi:10.1073/pnas.0706079105PubMedCentralPubMedCrossRef

Tanaka JW (2001) The entry point of face recognition: evidence for face expertise. J Exp Psychol Gen 130:534-543. doi:10.1037/0096-3445.130.3.534PubMedCrossRef

Taylor C, Schloss K, Palmer SE, Franklin A (2013) Color preferences in infants and adults are different. Psychon Bull Rev 20:916-922. doi:10.3758/s13423-013-0411-6PubMedCrossRef

Zajonc RB (1968) Attitudinal effects of mere exposure. J Pers Soc Psychol Monogr 9:11-27. doi:10. $\underline{1037 / h 0025848}$ 